\begin{tabular}{lccccc} 
RENAL & & 3393 & $13,4 \%$ & 576 & $13,8 \%$ \\
ICC* & 3651 & $14,4 \%$ & 501 & $12,0 \%$ \\
IHM & & 1035 & $4,1 \%$ & 156 & $3,7 \%$ \\
Cancer $^{*}$ & & 1177 & $4,6 \%$ & 131 & $3,1 \%$ \\
ECV $^{*}$ & & 1030 & $4,1 \%$ & 123 & $2,9 \%$ \\
EPOC & 736 & $2,9 \%$ & 112 & $2,7 \%$ \\
ION $^{*}$ & & 431 & $1,7 \%$ & 41 & $1 \%$ \\
CCI $^{*}$ & $\quad$ Without & 11943 & $47,0 \%$ & 2065 & $49,3 \%$ \\
& Comorbidities & & & & \\
& & 12355 & $48,7 \%$ & 1977 & $47,2 \%$ \\
& 2 Comorbidities & 1089 & $4,3 \%$ & 147 & $3,5 \%$ \\
Mortality $^{*}$ & 2 Comorbidities & 1618 & $6,4 \%$ & 177 & $4,2 \%$ \\
\hline
\end{tabular}

*Statistically significant differences $(P<0.05)$ DM2: Diabetes mellitus Type 2 PMR: Polymyalgia rheumatica ICC: Congestive heart failure IHM: Ischemic Heart Disease ECV: Cerebrovascular disease ION: ischemic optic neuropathy $\mathrm{CCI}$ : Charlson Index of Comorbidities

Conclusion: The patients with GCA with PMR represent a subtype of GCA with lower risk of stroke, ischemic optic neuropathy and mortality than patients with ACG without associated PMR.

Disclosure of Interests: : None declared

DOI: 10.1136/annrheumdis-2019-eular.3368

\section{THU0296 CORRELATIONS AMONG VASCULAR ULTRASOUND ABNORMALITIES OF THE TEMPORAL AND LARGE ARTERIES IN GIANT CELL ARTERITIS}

Michael Diiorio ${ }^{1}$, Piotr Sobieszczyk ${ }^{2}$, Julia Ford ${ }^{1}$, William Docken ${ }^{1}$, Sara Tedeschi ${ }^{1}$. ${ }^{1}$ Brigham and Women's Hospital, Division of Rheumatology, Allergy and Immunology, Boston, United States of America; ${ }^{2}$ Brigham and Women's Hospital, Division of Cardiovascular Medicine, Boston, United States of America

Background: Vascular ultrasound (VUS) is a sensitive and specific method for evaluating giant cell arteritis (GCA). Understanding patterns of temporal artery (TA) and large artery involvement may be important for aiding in diagnosis.

Objectives: To determine correlations among VUS abnormalities in patients with GCA.

Methods: We performed a retrospective study among 503 patients that underwent VUS to evaluate suspected or known GCA at an academic medical center, 2013-2017. Demographics, clinical features, VUS reports, and pathology data were extracted through electronic medical record review. Trained cardiovascular ultrasonographers imaged the right and left temporal, common carotid (CCA), subclavian (SCA), and axillary (AXA) arteries. The superficial temporal arteries (STA) and their frontal (TA-F) and parietal (TA-P) branches were abnormal if halo sign or hyperechoic wall thickening was present; the CCA, SCA and AXA were abnormal if those findings and/or stenosis or occlusion were present. Cardiovascular medicine physicians trained in VUS interpreted studies as acute arteritis, no arteritis, or hyperechoic wall thickening without acute arteritis. Among patients diagnosed with GCA by the treating physician $(n=139)$, we determined correlations between abnormal vessels using phi coefficients ( $\phi)$. We created composite variables representing each TA and its branches, and the left and right CCA, SCA, and AXA to test correlations between temporal and large arteries. Significance was determined using Fisher's exact test with Bonferroni correction.

Results: Among 139 patients, 50\% were diagnosed before VUS (median disease duration 17 months). Median age at VUS was 73 years; $75 \%$ were female and $93 \%$ White. Forty patients $(29 \%)$ had TA biopsy-proven GCA; an additional $14(10 \%)$ had biopsy-proven giant cell aortitis. Prevalence of abnormalities of TAs and large arteries were similar $(20 \%$ of right and $27 \%$ of left TAs; $20 \%$ of right and $24 \%$ of left large arteries). VUS was consistent with acute arteritis in $32 \%$, no arteritis in $53 \%$, and hyperechoic without acute arteritis in $15 \%$. Abnormalities in the STA, TA$F$, and TA-P were moderately correlated ipsilaterally $(\phi=0.46-0.62$, $p<0.0001)$ and contralaterally $(\phi=0.51-0.58, p<0.0001)$ (Table). Abnormalities in the AXA and SCA were strongly correlated with the ipsilateral and contralateral AXA and SCA $(\phi=0.64-0.82, \quad p<0.0001)$. Correlation between right and left TAs was moderate $(\phi=0.59, p<0.0001)$, while a stronger relationship was found between right and left large arteries $(\phi=$ $0.77, p<0.0001)$. Abnormalities in TAs were not correlated with large arteries ( $\phi \approx 0$ for all comparisons; not significant).

Conclusion: We observed moderate correlations of TA branches and strong correlations of large artery branches on VUS among GCA patients. Abnormalities in the TAs and large arteries were not correlated, underscoring the importance of scanning both groups of vessels with VUS.

\begin{tabular}{|c|c|c|c|}
\hline Artery & Correlated Artery & $\phi$ & $p$ \\
\hline \multirow[t]{4}{*}{$\overline{\text { R STA }}$} & R TA-F & 0.60 & $\dagger$ \\
\hline & R TA-P & 0.62 & $\dagger$ \\
\hline & L STA & 0.58 & $\dagger$ \\
\hline & L TA-F & 0.51 & $\dagger$ \\
\hline \multirow[t]{2}{*}{ R TA-F } & R TA-P & 0.58 & $\dagger$ \\
\hline & L STA & 0.48 & $\dagger$ \\
\hline R TA-P & L TA-P & 0.51 & $\dagger$ \\
\hline L STA & L TA-F & 0.55 & $\dagger$ \\
\hline L TA-F & L TA-P & 0.46 & $\dagger$ \\
\hline R CCA & L CCA & 0.66 & $\dagger$ \\
\hline \multirow[t]{3}{*}{ R AXA } & R SCA & 0.81 & $\dagger$ \\
\hline & L AXA & 0.82 & $\dagger$ \\
\hline & L SCA & 0.81 & $\dagger$ \\
\hline L AXA & L SCA & 0.69 & $\dagger$ \\
\hline \multirow[t]{2}{*}{ R SCA } & L AXA & 0.64 & $\dagger$ \\
\hline & L SCA & 0.73 & $\dagger$ \\
\hline \multirow[t]{3}{*}{$\begin{array}{l}\mathrm{R} \text { temporal } \\
\text { composite }\end{array}$} & $\begin{array}{l}\mathrm{L} \text { temporal } \\
\text { composite }\end{array}$ & 0.59 & $\dagger$ \\
\hline & $\mathrm{R}$ large composite & -0.07 & NS \\
\hline & L large composite & -0.07 & NS \\
\hline \multirow{2}{*}{$\begin{array}{l}\text { L temporal } \\
\text { composite }\end{array}$} & $\mathrm{R}$ large composite & 0.06 & NS \\
\hline & L large composite & 0.05 & NS \\
\hline $\mathrm{R}$ large composite & L large composite & 0.77 & $\dagger$ \\
\hline
\end{tabular}

Temporal composite: abnormal superficial TA, TA-frontal branch, and/or TA-parietal branch. Large composite: abnormal common carotid, subclavian, and/or axillary artery. NS: not significant

$\dagger p<0.0001$

Disclosure of Interests: None declared

DOI: 10.1136/annrheumdis-2019-eular.2902

\section{THU0297 STEROID TREATMENT MAY IMPROVE ARTERIAL STIFFNESS IN PATIENTSWITH POLYMYALGIA RHEUMATICA AND GIANT CELL ARTERITIS: A PROSPECTIVE COHORT STUDY}

Amir Emamifar ${ }^{1,2,3}$, Torkell Ellingsen ${ }^{4}$, Søren Hess ${ }^{5,6}$, Per Syrak Hansen ${ }^{2}$, Inger Marie Jensen Hansen ${ }^{3}$, Peter Thye-Rønn ${ }^{1,2}$. ${ }^{1}$ University of Southern Denmark, Clinical Research, Odense, Denmark; ${ }^{2}$ Svendborg Hospital, Diagnostic Center Svendborg, Denmark; ${ }^{3}$ Svendborg Hospital, Rheumatology, Svendborg, Denmark; ${ }^{4}$ Odense University Hospital, Rheumatology, odense, Denmark; ${ }^{5}$ Hospital of Southwest Jutland, Radiology and Nuclear Medicine, Esbjerg, Denmark;

${ }^{6}$ University of Southern Denmark, Regional Health Research, Odense, Denmark

Background: Polymyalgia rheumatica (PMR)/Giant cell arteritis (GCA), common inflammatory diseases, are associated with increased risk of aortic stiffness, possibly due to an inflammatory process. ${ }^{1,2}$

Objectives: To evaluate the effect of steroid treatment on aortic stiffness in patients with PMR/GCA.

Methods: This is an ongoing 1-year prospective cohort study. 37 consecutive patients with newly diagnosed PMR/GCA were included. Aortic pulse wave velocity (PWV) and aortic augmentation index normalized to heart rate of 75 beats per minute (Alx@75) were measured at baseline and subsequently at $1^{\text {st }}$ and $4^{\text {th }}$ months of treatment initiation with oral prednisolone.

Results: Of 37 patients, 3 pts. were excluded from the study because of lack of interest or a change in the initial diagnosis. Of all included pts. $61.8 \%$ were female and mean age was 71 (69-74) years. At diagnosis 24 pts. presented with pure PMR symptoms, 2 pts. with pure cranial GCA, 8 pts.with concurrent PMR and GCA. The mean of erythrocyte sedimentation rate (ESR) and C-reactive protein (CRP) at baseline were $62.3 \pm 22.9$ and $46.4 \pm 43.7$, respectively. Alx@75 was significantly

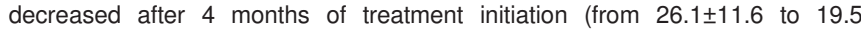
$\pm 11.5, p=0.016$ ). Though aortic PWV was decreased after 4 months of treatment initiation (from $12.3 \pm 2.4$ to $11.6 \pm 2.4$ ), the results was not statistically significant $(p=0.070)$. We did not find any significant changes at $1^{\text {st }}$ month, nor in aortic PWV neither in Alx@75.

Conclusion: Steroid treatment may improve arterial stiffness in PMR/GCA patients due to suppression of the inflammatory process. However this is a time consuming effect and may not be seen as early as the first month.

\section{REFERENCES:}

[1] Emamifar A, et al. Polymyalgia rheumatica and giant cell arteritis-three challenges-consequences of the vasculitis process, osteoporosis, and 
malignancy: A prospective cohort study protocol. Medicine (Baltimore). 2017;96(26):e7297.

[2] Schillaci G, et al. Aortic stiffness is increased in polymyalgia rheumatica and improves after steroid treatment. Ann Rheum Dis 2012;71:1151-6.

Disclosure of Interests: None declared

DOI: 10.1136/annrheumdis-2019-eular.4987

\section{THU0298 INITIAL MANIFESTATIONS AND OUTCOME OF PATIENTS WITH INCOMPLETE BEHÇET'S SYNDROME}

Sinem Nihal Esatoglu ${ }^{1}$, Seyda Bilgin ${ }^{2}$, Cem Sulu $^{2}$, Vedat Hamuryudan $^{3}$, Zekayi Kutlubay ${ }^{4}$, Serdal Ugurlu ${ }^{3}$, Emire Seyahi ${ }^{3}$, Melike Melikoglu $^{3}$, Izzet Fresko ${ }^{3}$ Sebahattin Yurdakul ${ }^{3}$, Hasan Yazici ${ }^{3}$, Gulen Hatemi ${ }^{3} .{ }^{1}$ Istanbul UniversityCerrahpasa, Cerrahpasa Medical Faculty, Department of Internal Medicine, Division of Rheumatology, Istanbul, Turkey, ${ }^{2}$ Istanbul University-Cerrahpasa, Cerrahpasa Medical Faculty, Department of Internal Medicine, Istanbul, Turkey, ${ }^{1}$ Istanbul University-Cerrahpasa, Cerrahpasa Medical Faculty, Department of Internal Medicine, Division of Rheumatology, Istanbul, Turkey; ${ }^{4}$ Istanbul UniversityCerrahpasa, Cerrahpasa Medical Faculty, Department of Dermatology, Istanbul, Turkey

Background: Behçet's syndrome (BS) has a heterogeneous expression involving many organ systems and is diagnosed by recognizing the coexisting manifestations. Several patients initially have few of these manifestations and do not fulfill the International Study Group (ISG) criteria When these are major organ manifestations, failure to recognize BS and treat promptly may lead to permanent damage in these organs.

Objectives: The aim of this study is to highlight the magnitude of this problem by surveying the frequency, presentation patterns and outcome of patients who did not fulfill ISG criteria when they presented to our clinic, but were followed and treated for manifestations strongly suggesting BS.

Methods: We conducted a retrospective chart review of all BS patients who were registered between 2003 and 2008. Among these 2385 patients, $199(8 \%)$ BS patients who did not fulfill ISG criteria at their initial visit were included in this study. Patients were called and a standard form was used for collecting demographic characteristics, BS manifestations at initial visit and during follow-up and treatment.

Results: Among the 199 patients (M/W: 90/109, mean age: $34 \pm 11$ years) who did not fulfill ISG criteria when they presented to our clinic $70(35 \%)$ had major organ involvement. The types of major organ involvement that led to a diagnosis of BS at initial visit despite not fulfilling ISG criteria were eye involvement in 37, vascular involvement in 29 (venous thrombosis in 22, arterial aneurysms in 7), nervous system involvement in 3 and gastrointestinal (Gl) involvement in 1 patient. Thirtyfive patients $(18 \%)$ had a family history of BS

Of 199 patients, 167 had at least one more visit with a median follow-up of 11 years (IQR: 7-12). We were able to contact 116 of these patients and saw that 53 had fulfilled ISG criteria in the meantime. Among the 51 patients that we were not able to contact, 17 had fulfilled criteria while they were being followed in our clinic. Thus, a total of $70(42 \%)$ patients fulfilled ISG criteria after a median follow-up of 1.5 years (IQR: 1-4.25). All but 2 patients who developed eye involvement during the follow-up had fulfilled ISG criteria with a new mucocutaneous manifestation After a median follow-up of 4 years (IQR: 1-7), 23 (14\%) patients had developed at least one non-criteria BS manifestation, including vascular involvement in 10, arthritis in 13, neurologic involvement in 2 patients and $\mathrm{Gl}$ involvement in 1 patient.

Among the 81 patients who developed at least one new manifestation, $16(20 \%)$ were under immunosuppressive or interferon-alpha treatment at the time they developed their new manifestation. The remaining 65 patients had only received colchicine.

Among the 29 patients who had vascular involvement at initial visit, 7 (24\%) had a vascular relapse at different vascular site.

Three patients had another rheumatologic diagnosis (ankylosing spondylitis, seropositive rheumatoid arthritis and sarcoidosis) at the end of the follow-up.

Conclusion: In our 10-year follow-up cohort study, $42 \%$ of the 167 incomplete BS patients had fulfilled ISG criteria within a median duration of 1.5 years. Sixty-two (37\%) of these had major organ involvement that could have caused severe morbidity and mortality if the diagnosis of BS was missed and patients were untreated.

Disclosure of Interests: : Sinem Nihal Esatoglu: None declared, Seyda Bilgin: None declared, Cem Sulu: None declared, Vedat Hamuryudan
Consultant for: Abbvie, Amgen, BMS, Jansen, MSD, Pfizer, UCB, Speakers bureau: Abbvie, Amgen, BMS, Jansen, MSD, Pfizer, UCB, Zekayi Kutlubay: None declared, Serdal Ugurlu: None declared, Emire Seyahi: None declared, Melike Melikoglu: None declared, Izzet Fresko: None declared, Sebahattin Yurdakul: None declared, Hasan Yazici: None declared, Gulen Hatemi Consultant for: Abbvie, Amgen, BMS, Janssen, MSD, Pfizer, UCB, Speakers bureau: Abbvie, Amgen, BMS, Jansen, MSD, Pfizer, UCB,

DOI: 10.1136/annrheumdis-2019-eular.4287

\section{THU0299 PATIENT GLOBAL ASSESSMENT OF DISEASE ACTIVITY IN BEHÇET'S SYNDROME: A MULTICENTER STUDY}

Alberto Floris ${ }^{1}$, Matteo Piga ${ }^{1}$, Gerard Espinosa ${ }^{2}$, Nikolaos Kougkas $^{3}$, Andrea Lo Monaco $^{4}$, Giuseppe Lopalco ${ }^{5}$, Ida Orlando ${ }^{6}$, Vittorio Pirani ${ }^{7}$,

Ernestina Santos ${ }^{8,9}$, Luísa Serpa Pinto ${ }^{10}$, George Bertsias ${ }^{3}$, Luca Cantarini ${ }^{6}$, Alberto Cauli ${ }^{1}$, Ricard Cervera ${ }^{2,2}$, João Correia ${ }^{10}$, Marcello Govoni ${ }^{4}$, Florenzo lannone ${ }^{5}$, Ana Martins Da Silva ${ }^{8,9}$, Piergiorgio Neri ${ }^{11}$

Carlos Vasconcelos ${ }^{9}$, Alessandro Mathieu ${ }^{1} .{ }^{1}$ University of Cagliari, Rheumatology, Monserrato, Italy; ${ }^{2}$ University of Barcelona, Department of Autoimmune Diseases, Barcelona, Catalonia, Spain; ${ }^{3}$ University of Crete, Rheumatology, Clinical Immunology and Allergy, Heraklion, Greece; ${ }^{4}$ University of Ferrara, Rheumatology, Ferrara, Italy; ${ }^{5}$ University of Bari, Rheumatology, Bari, Italy; ${ }^{6}$ University of Siena, Rheumatology, Siena, Italy; ${ }^{7}$ Università Politecnica delle Marche, Ophthalmology, Ancona, Italy; ${ }^{8}$ Centro Hospitalar do Porto/Hospital de Santo António, Neurology, Porto, Portugal; ${ }^{9}$ University of Porto, UMIB Abel Salazar Biomedical Sciences Institute, Porto, Portugal; ${ }^{10}$ Hospital Santo Antonio Centro Hospitalar do Porto, Unidade de Imunologia Clinica, Unidade de Imunologia Clinica, Porto, Portugal;

${ }^{11}$ Cleveland Clinic Abu Dhabi, Eye Institute, Abu Dhabi, United Arab Emirates

Background: Disease activity evaluation is mandatory according to the OMERACT Core Set of Domains for Behçet's Syndrome (BS). Poor data are available on Patient (PtGA) and physician (PGA) global assessment of disease activity in BS.

Objectives: To assess PtGA performance in patients with BS and how different disease manifestations influence the patient and physician's perception of disease activity.

Methods: A multicenter cross-sectional cohort of consecutive BS patients was enrolled. Disease activity was evaluated by PtGA, PGA and the Behçet's disease current activity form (BDCAF). PtGA and PGA were assessed trough a single question ("How active was BS during the last week?") in a $10-\mathrm{cm}$ visual analogic scale. Health related quality of life (HRQoL) perception was evaluated by the Physical Component Summary (PCS) and the Mental Component summary (MCS) of the SF36v2 questionnaire.

Correlation between PtGA, PGA, BDCAF and between them and SF36v2 component summaries was assessed trough the Spearman's correlation coefficient (rho). Two separated multiple regression models were built to measure the independent effect ( $\beta$ regression) of each active clinical manifestation on PtGA and PGA

Results: Overall, 226 BS patients from 5 Mediterranean Countries were enrolled. Out of them, $111(49.1 \%)$ were males and the median (IQR) age and disease duration were 46.9 (35.6-55.2) and 11.7 (5.9-20.8) years, respectively.

The median (IQR) value of PtGA, PGA and BDCAF were 2.0 (0.3-5.0) $1.0(0.0-3.0)$ and $3.0(0.0-5.0)$, respectively. PtGA significantly correlated (Figure) with both PGA (rho 0.759. $p<0.001$ ) and BDCAF (rho 0.523. p $<0.001$ ), in support of its construct validity. PtGA scores were significantly higher than PGA with a mean (SD) difference of 0.8 (1.8), suggesting a different weighing of disease activity between patients and physicians. Indeed, PtGA and PGA were differently influenced by active clinical manifestations (Table). PtGA was mainly dependent on active mucocutaneous ( $\beta$ 0.243), gastrointestinal $\left(\beta\right.$ 0.216) and ocular $\left(\begin{array}{ll}\beta & 0.209\end{array}\right)$ involvement whereas a major effect of ocular manifestations was observed in PGA ( $\beta$ 0.378). PtGA was correlated with worse HRQoL perception measured by PCS (rho -0.476. $p>0.001$ ) and MCS (rho -451 $p>0.001$ ).

Conclusion: PtGA seems to be a valid outcome measure in BS and should be considered as a separate outcome measure or better be included in composite index for overall disease activity evaluation.

Abstract THU299 - Table 1

PtGA 